\title{
Unraveling exciton dynamics in amorphous silicon dioxide: Interpretation of the optical features from 8 to $11 \mathrm{eV}$
}

\author{
Eleonora Vella, Fabrizio Messina, Marco Cannas, and Roberto Boscaino \\ Dipartimento di Scienze Fisiche ed Astronomiche, Universitàdi Palermo, Via Archirafi 36, I-90123 Palermo, Italy
}

(Received 10 September 2010; revised manuscript received 2 March 2011; published 9 May 2011)

\begin{abstract}
We introduce a model comprehensively describing the optical features of amorphous silicon dioxide $\left(a-\mathrm{SiO}_{2}\right)$ in the spectral range from $\sim 8$ up to $\sim 11 \mathrm{eV}$. Our model is grounded on the critical analysis of the temperature dependence of Kramers-Kronig-derived absorption spectra in the range from $\sim 8$ up to $\sim 17.5 \mathrm{eV}$, together with the features of the Urbach absorption tail and of self-trapped exciton emission. In a paper we recently published [Phys. Rev. Lett. 105, 116401 (2010)] we showed the 10.4-eV resonance in the absorption spectra to feature a close Lorentzian line shape, thus implying a delocalized nature for excitons in $a-\mathrm{SiO}_{2}$. Here we provide estimations of the main parameters ruling exciton dynamics in $\mathrm{SiO}_{2}$, such as the energy of the mean lattice vibrational mode coupled to excitons $\left(\hbar \omega_{0}=0.083 \mathrm{eV}\right)$, the half width of the excitonic energy band $(B \sim 2 \mathrm{eV})$, the root-mean-square amplitude of site-to-site energy fluctuations of exciton energy $(D \sim 0.7 \mathrm{eV})$, and the exciton-phonon coupling constant $(g \sim 2.1)$. The quantum yield of excitonic emission $\left(\eta \sim 10^{-3}\right)$ at $T=10 \mathrm{~K}$ in $a-\mathrm{SiO}_{2}$ is determined as well. Our model suggests that $a-\mathrm{SiO}_{2}$ features an indirect gap near $\sim 9$ $\mathrm{eV}$ and a direct one near $\sim 11 \mathrm{eV}$, and allows a coherent description of the properties of the intrinsic Urbach absorption tail. The latter results are satisfactorily explained as arising from the momentary self-trapping of the 10.4-eV exciton. As far as near-edge absorption properties are concerned, our model places $\mathrm{SiO}_{2}$ in the wider context of wide-band-gap solids, such as LiF or NaF, where excitons are weakly scattered, but strongly coupled to phonons. On the whole, the present study shows that exciton dynamics accounts for all optical properties of $a-\mathrm{SiO}_{2}$ from $\sim 8$ up to $\sim 11 \mathrm{eV}$.
\end{abstract}

DOI: 10.1103/PhysRevB.83.174201

PACS number(s): 71.35.Cc, 71.23.-k, 78.40.Pg

\section{INTRODUCTION}

Amorphous silicon dioxide $\left(a-\mathrm{SiO}_{2}\right)$, or silica, is a solid of primary technologic importance, particularly useful in optical applications requiring a high UV transparency. As a consequence, a strong research effort is currently devoted to further improve the optical properties of this material in view of prospective vacuum UV (VUV, $\lambda<200 \mathrm{~nm}$ ) applications. ${ }^{1-5}$ Moreover, $a-\mathrm{SiO}_{2}$ is an important model system to investigate the physical properties of glassy solids; this study is often aided by a comparison with $\alpha$-quartz $\left(c-\mathrm{SiO}_{2}\right)$, the most common crystalline polymorph of silicon dioxide. Some of the basic open problems in the physics of glassy $\mathrm{SiO}_{2}$ concern the fundamental absorption edge arising from the electronic transition from the valence to the conduction band. From an experimental point of view, this issue is usually investigated by the combined use of different techniques. Optical absorption spectroscopy allows to characterize the region in which the absorption coefficient $\alpha$ of a bulk solid is smaller than $10^{3}-$ $10^{4} \mathrm{~cm}^{-1}$ and, in systems such as $\mathrm{SiO}_{2}$, it is affected by additional technical complications since the absorption edge falls in the VUV spectral region. In this portion of the spectrum, starting from $\sim 8 \mathrm{eV}$, both $a-\mathrm{SiO}_{2}$ and $c-\mathrm{SiO}_{2}$ feature the exponential absorption region usually referred to as the Urbach tail. ${ }^{6}$ At shorter wavelengths, $\alpha$ becomes exceedingly high to be estimated via standard spectrophotometric measurements in bulk materials, and thus the absorption spectrum can be only indirectly inferred by a Kramers-Kronig (K-K) analysis of reflectivity data. Although straightforward in principle, this approach can be quite tricky due to the need for reliable measurements in a sufficiently extended range, obtained by use of a suitable wide-band VUV optical source. This has limited the number of K-K investigations on silica and quartz to date, ${ }^{7-12}$ and consequently hindered the comprehensive understanding of several features, even fundamental, of aboveedge absorption in $\mathrm{SiO}_{2}$-based systems.

The exact position and the characteristics of the fundamental electronic transition in $\mathrm{SiO}_{2}$ are still debated, even though there have been many experiments devoted to their clarification. Several experimental results have led to an estimate of the band-to-band separation of $c-\mathrm{SiO}_{2}$ and $a-\mathrm{SiO}_{2}$ to be $\sim 9 \mathrm{eV},{ }^{10,12-18}$ including the detection of photoconductivity starting from $8.9 \mathrm{eV},{ }^{13,14}$ the $8.3-\mathrm{eV}$ photoexcitation threshold of the 2.8-eV luminescence attributed to self-trapped excitons, ${ }^{17}$ and the detection in K-K-transformed reflectivity spectra of a region in which absorption is proportional to $\left(E-E_{g}\right)^{3 / 2}$, with $E_{g}=9.3 \mathrm{eV} \cdot{ }^{10,18}$ On the other side, some studies questioned these findings, by reporting that photoconductivity and photoemission signals markedly increase only above $11 \mathrm{eV}$, this being accompanied by qualitative variations of the photoelectron energy distribution curve. ${ }^{19-22}$ Hence, it was argued that the photoemission signal originally observed below $11 \mathrm{eV}$ was a spurious effect not related to actual band-to-band excitation of the material, while the actual band gap of $\mathrm{SiO}_{2}$ was proposed to lay at $\sim 11 \mathrm{eV}^{21}$ Finally, based on both computational ${ }^{23,24}$ and experimental results ${ }^{17}$ some authors proposed the band gap at $\sim 9 \mathrm{eV}$ in $\mathrm{SiO}_{2}$ to be indirect, with the threshold for direct band-to-band transitions located at $\sim 11 \mathrm{eV}$.

The lowest-energy peak in the reflectivity spectra of $c$ $\mathrm{SiO}_{2}$ and $a-\mathrm{SiO}_{2}$ is at $10.3 \mathrm{eV}$, with qualitatively similar characteristics in both systems, ${ }^{12,15,22,25}$ and it corresponds to a peak in the absorption spectra at $\sim 10.4 \mathrm{eV}$. This band was promptly attributed to an excitonic transition based on 
its temperature dependence, as observed in the 300-600 K interval by measurements in $c$-SiO ${ }_{2} \cdot{ }^{8,15,25,26}$ Photoluminescence measurements upon above-gap excitation of $c-\mathrm{SiO}_{2}$ and $a-\mathrm{SiO}_{2}$ doped with luminescent impurities have led some authors to distinguish different types of excitons based on their different mobility and energy-transfer properties. ${ }^{19,21,22}$

We have recently shown the $10.4-\mathrm{eV}$ peak in $a-\mathrm{SiO}_{2}$ to have a Lorentzian line shape, thus implying that excitons retain their delocalized properties albeit thermal and structural disorder, which is typical of an amorphous material. ${ }^{27}$ In the present study we deepen this interpretation of exciton physics in $\mathrm{SiO}_{2}$ by providing a comprehensive discussion of excitonic absorption and emission founded on reflectivity and photoluminescence measurements. The main goal of the present work is introducing, via a critical analysis of existing literature data and comparison with our own results, a self-consistent description encompassing the features of the excitonic peaks and those of the low-energy Urbach absorption tail. This achievement is interesting for at least a couple of reasons. First, our scheme allows to place the near-edge absorption properties of $a-\mathrm{SiO}_{2}$ in the wider context of excitonic properties in wide-band-gap solids, showing that the material can be likened to systems such as $\mathrm{LiF}$, in that excitons are weakly scattered by, but strongly coupled to, phonons. Second, our model sheds light on the properties of the Urbach absorption edge in $a-\mathrm{SiO}_{2}$, which remains one of the most elusive aspects among the spectroscopic characteristics of the material.

\section{THEORETICAL DESCRIPTION OF EXCITONIC OPTICAL PROPERTIES}

In the absence of exciton-phonon interaction, an excitonic absorption line in a crystal would be infinitely sharp and located at the energy $E_{P}^{0}$ corresponding to the creation of the exciton with wave number $K=0$, in order to fulfill the conservation of crystalline momentum in photon absorption. The well-established theory by Toyozawa and co-workers describes under certain basic assumptions the modifications of exciton optical properties arising from the quasiparticle interaction with the phonon bath. ${ }^{31,32}$ It turns out that when exciton-phonon coupling (EPC) is taken into account, the resulting absorption line shape is basically governed by the competition between the mobile nature of excitons and their tendency to localize in the lattice due to EPC. The former is measured by the half width $B$ of the energy band of excitons, proportional to the site-to-site transfer rate, while the latter is measured by the root-mean-square amplitude $D$ of the fluctuations of exciton energy due to the thermal activated vibrations of the lattice. Theory predicts that if the ratio $D / B \ll 1$ and the mobile nature of the exciton is thus prevailing (weak scattering limit), the exciton absorption line shape is Lorentzian: ${ }^{31-33}$

$$
\alpha(E, T)=\alpha_{0}\left[1+\left(\frac{E-\left[E_{P}^{0}+\Delta_{0}(T)\right]}{\Gamma_{0}(T)}\right)^{2}\right]^{-1} .
$$

While the delocalized nature of exciton states is not substantially altered by weak phonon scattering, the Lorentzian broadening is ultimately due to the fact that the $K=0$ exciton acquires a finite lifetime due to phonon-induced scattering toward $K \neq 0$ levels. This situation can occur either if EPC is inefficient (small $D$ ) or if excitons are mobile enough (large $B$ ) to compensate for its effects. In this case, the peak position $E_{P}^{0}$ is perturbed by the complex self-energy $\Delta_{0}(T)+i \Gamma_{0}(T)$ of the exciton in the phonon field, giving rise to a temperaturedependent shift $\left[\Delta_{0}(T)\right]$ and broadening $\left[\Gamma_{0}(T)\right]$ of the exciton line ( $i$ is the imaginary unit). The predicted full width at half maximum $(\mathrm{FWHM})=2 \Gamma_{0}(T)$ can be expressed as $c D^{2} / B$, where the adimensional proportionality coefficient $c$ is the parameter most affected by the details involved in theoretical modeling of exciton-phonon interaction and by the detailed features of the exciton band. If the $K=0$ level is neither at the bottom nor at the top of the excitonic band, the first-order value of $c$ turns out to be $\sim \pi{ }^{34}$ The quantities $D, \Delta_{0}(T)$, and $\Gamma_{0}(T)$ are expected to increase with growing temperature due to progressive thermal activation of the lattice. Indicating with $\hbar \omega_{0}$ the energy of the mean phonon mode coupled to the exciton, the predicted temperature dependencies are in the form

$$
\frac{\Delta_{0}(T)}{\Delta_{0}^{0}}=\frac{\Gamma_{0}(T)}{\Gamma_{0}^{0}}=\frac{D^{2}}{\hbar \omega_{0} E_{\mathrm{LR}}}=\operatorname{coth}\left(\frac{\hbar \omega_{0}}{2 k_{B} T}\right),
$$

where the parameter $E_{\mathrm{LR}}$ can be interpreted as the lattice relaxation energy after the creation of an exciton due to vibrational decay within the excited electronic state.

As $D$ grows with temperature, the effect of EPC on the excitonic absorption line increases until it cannot be considered anymore as a small perturbation affecting the delocalized exciton states. In particular, for large thermal or structural disorder, or for small $B$, that $D / B \gg 1$ (strong scattering limit), the model foresees the overall exciton line shape to be Gaussian, with $\sim 2.35$ D FWHM. ${ }^{31-33}$ This case corresponds to a completely localized, defectlike exciton, whose absorption line basically reproduces the density of states, whose energy thermally fluctuates with Gaussian statistics from site to site.

It is worth noting that the transition between delocalized $(D \ll B)$ and localized $(D \gg B)$ excitons must be regarded as a gradual one, and as a function of increasing thermal disorder. On the other side, the relaxation pathways of the exciton returning to the ground state after excitation are predicted to feature an almost abrupt transition between two situations: excitons which go back to the ground state by emitting a resonant sharp emission line still associated to a free exciton (FE) and systems emitting the typical broad Stokes-shifted band due to self-trapped excitons (STEs). The transition is controlled by the value of the so-called EPC constant $g=E_{\mathrm{LR}} / B$ : If $g>1$ (strong EPC), exciton emission is of the self-trapped type at energy $\sim E_{P}^{0}-2 E_{\mathrm{LR}}$, while $g<2 / 3$ (low EPC) leads to FE emission without a significant Stokes shift from the absorption line..$^{32,34-36}$ The interval $2 / 3<g<1$ allows for the coexistence of STE and FE emissions. The strong EPC condition is associated with the existence of an energetically stable STE state to which the exciton can relax upon a lattice rearrangement. Since the boundary between weak and strong scattering $(D / B=1)$ is different from that between weak and strong coupling $(g=1)$, a strong EPC does not necessarily imply strong scattering and vice versa: In particular, values of $B, E_{\mathrm{LR}}$, and $D$ are possible for which strong coupling and weak scattering coexist. In this situation, 
the exciton behaves as a nearly free quasiparticle until it finally surmounts the potential barrier to become self-trapped. ${ }^{34}$

Finally, Toyozawa's model is even able to successfully predict the existence of the exponential absorption tail, experimentally observed in a wide variety of materials, known as the Urbach rule, ${ }^{32,37}$ and usually treated only from a phenomenological point of view. Actually, in the framework of Toyozawa's theory, such a tail naturally arises in the far low-energy wing of a weak scattered Lorentzian excitonic absorption peak. In fact, even in the weak scattering case, EPC induces the appearance of localized states below the bottom of the excitonic band, which can be regarded as temporary STE states showing up from time to time due to sufficiently great local lattice deformations. The existence of localized states below delocalized ones in the thermally disordered lattice can be actually considered as a form of Anderson localization. ${ }^{32}$ Thus, while the main Lorentzian absorption band is associated with the delocalized states, excitation of the localized states of lower energy gives rise to an absorption wing which has been numerically predicted to be exponential: ${ }^{32,36}$

$$
\alpha(E)=\alpha_{0} \exp \left(-\sigma \frac{E_{0}-E}{k_{B} T}\right),
$$

where $\sigma$ is expected to depend on temperature according to

$$
\sigma=\sigma_{0} \frac{2 k_{B} T}{\hbar \omega_{0}} \tanh \left(\frac{\hbar \omega_{0}}{2 k_{B} T}\right) .
$$

The parameter $\sigma_{0}$ controlling the slope of the exponential tail is related to the EPC constant, $\sigma_{0}=s / g$, where $s$ is a dimensionless parameter of the order of unity depending on geometrical factors such as lattice structure. ${ }^{32,36}$ This relation allows to estimate the EPC constant $g$ from absorption measurements in the region of the Urbach tail and thus to predict the existence of STE (if $g>1$ ) or FE (if $g<1$ ) emission. This prediction has been verified to be correct for a great number of materials. ${ }^{32}$

\section{SUMMARY OF THE EXPERIMENTAL RESULTS}

We briefly summarize some of the experimental results on $\mathrm{SiO}_{2}$ which our model is grounded on: These data include $\mathrm{K}-\mathrm{K}$-derived absorption spectra and photoluminescence emission under VUV excitation. We already reported part of the $\mathrm{K}-\mathrm{K}$-derived absorption data in a recently published paper ${ }^{27}$ and the reader may refer to that work for the description of the experimental setup and data analysis. ${ }^{27}$ The luminescence spectra we will report in the following were not presented before by us: Details concerning their acquisition are given at the end of this section.

Figure 1 shows the absorption coefficient of $a-\mathrm{SiO}_{2}$ and $c-\mathrm{SiO}_{2}$ in the range from 8 to $17.5 \mathrm{eV}$. The a-SiO $\mathrm{S}_{2}$ sample is a commercial type IV synthetic samples [commercial nickname: Suprasil F300, trademark of Heraeus Quarzglas (http://heraeus-quarzglas.com/en/home/Home.aspx), $\mathrm{OH}$ content $<1$ part per million in weight), whereas the $c-\mathrm{SiO}_{2}$ sample is a $z$-cut $\alpha$-quartz sample. The absorption spectra were obtained from the reflectivity spectra (not reported) using a K-K dispersion analysis. ${ }^{27}$ Four main peaks are present at $\sim 10.4, \sim 11.6, \sim 14.1$, and $\sim 17.3 \mathrm{eV}$. As can be seen, the absorption spectra of $a-\mathrm{SiO}_{2}$ are significantly affected

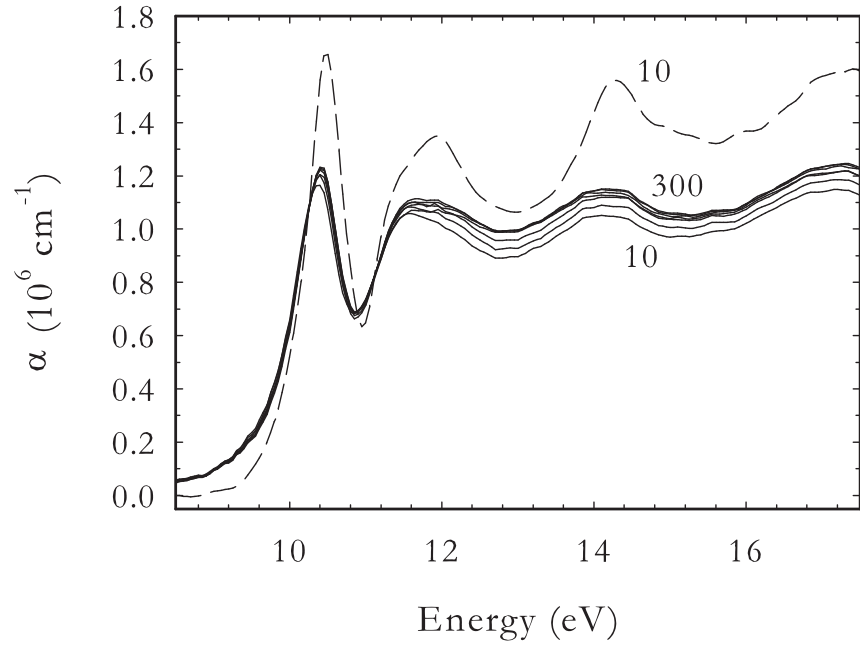

FIG. 1. K-K-derived absorption coefficient of $a-\mathrm{SiO}_{2}$ (solid lines) in the temperature range from 10 to $300 \mathrm{~K}$ in steps of $50 \mathrm{~K}$ and at $10 \mathrm{~K}$ for $c-\mathrm{SiO}_{2}$ (dashed line). Labels represent temperatures $(\mathrm{K})$ of the closest curve.

by temperature. In order to evidence the dependence of the features of the peaks on temperature, enlargements of both peaks at $\sim 10.4$ and $\sim 11.6 \mathrm{eV}$ are shown in Fig. 2. In Fig. 2(a) the absorption spectra in the range from 9 to $11 \mathrm{eV}$ are presented: With increasing temperature the peak at $\sim 10.4 \mathrm{eV}$ shifts toward lower energies. The enlargement in the region of the $\sim 10.5-\mathrm{eV}$ peak of the absorption spectrum of $c-\mathrm{SiO}_{2}$ at $10 \mathrm{~K}$ obtained from a K-K analysis is reported for comparison. The changes of the peak at $\sim 11.6 \mathrm{eV}$ are not monotonous with temperature, as evident from the enlargements of the spectra in the range from $\sim 11$ to $\sim 13 \mathrm{eV}$ shown in Figs. 2(b) and 2(c), where the absorption spectra from 300 to $150 \mathrm{~K}$ and from 150 to $10 \mathrm{~K}$ are reported, respectively. In the range from 300 to $150 \mathrm{~K}$ [Fig. 2(b)], absorption at $\sim 11.6 \mathrm{eV}$ decreases with increasing temperature, whereas in the range from 150 to $10 \mathrm{~K}$ [Fig. 2(c)] it grows with increasing temperature.

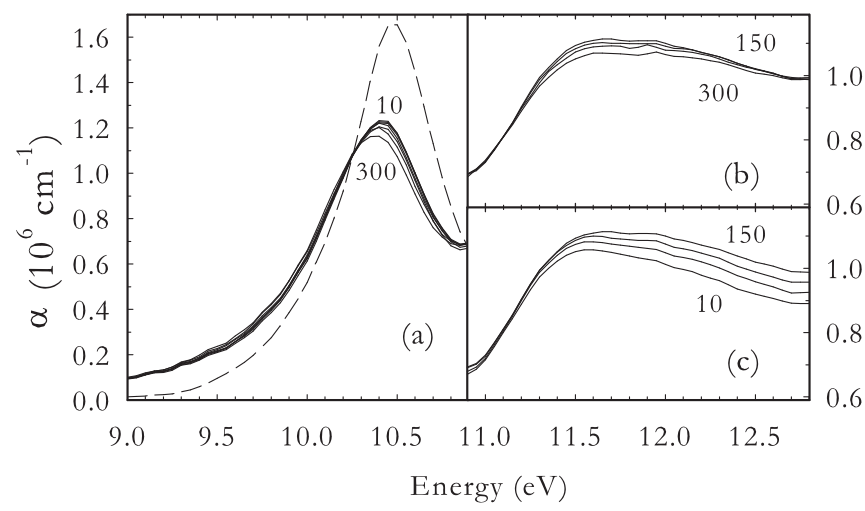

FIG. 2. (a) Enlargement of the absorption peak at $\sim 10.4 \mathrm{eV}$ from 10 to $300 \mathrm{~K}$ in steps of $50 \mathrm{~K}$ (full lines). Absorption coefficient of $c-\mathrm{SiO}_{2}$ at $10 \mathrm{~K}$ in the same spectral region (dashed line). Spectra are taken from Fig. 2(b) of Ref. 27. (b) Enlargement of the peak at $\sim 11.6 \mathrm{eV}$ in $a-\mathrm{SiO}_{2}$ from 300 to $150 \mathrm{~K}$ in steps of $50 \mathrm{~K}$. (c) Enlargement of the peak at $\sim 11.6 \mathrm{eV}$ in $a-\mathrm{SiO}_{2}$ from 150 to $10 \mathrm{~K}$ in steps of $50 \mathrm{~K}$. Labels represent temperatures $(\mathrm{K})$ of the closest curve. 


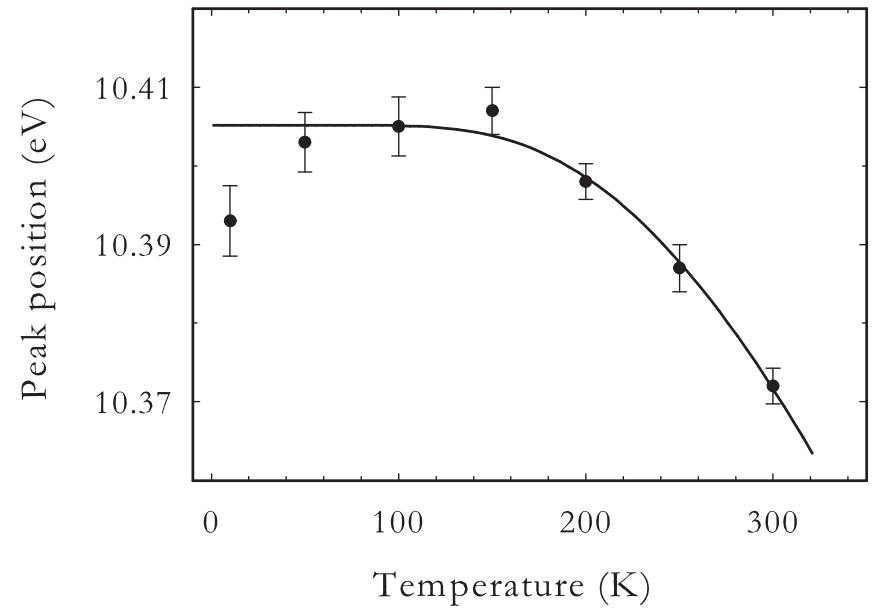

FIG. 3. (Color online) Position of the peak at $\sim 10.4 \mathrm{eV}$ in $a-\mathrm{SiO}_{2}$ as a function of temperature, as determined via a Lorentzian fit of the peak profiles. The full line is discussed in the text. Data are taken from inset (a) of Fig. 3 of Ref. 27.

As far as the two remaining peaks at $\sim 14.1$ and $\sim 17.3 \mathrm{eV}$ are concerned, the spectra in Fig. 1 show that in the range from 10 to $150 \mathrm{~K}$ their overall intensities increase, whereas for higher temperatures they do not change noticeably.

As was already discussed, ${ }^{27}$ the peak at $\sim 10.4 \mathrm{eV}$ in $a-\mathrm{SiO}_{2}$ turns out to have a very good Lorentzian profile at all temperatures. Figure 3 shows the position of the peak at $\sim 10.4 \mathrm{eV}$ as a function of temperature, as obtained by the Lorentzian fitting. The position of the peak remains approximately constant around a value of $\sim 10.41 \mathrm{eV}$ from 10 to $150 \mathrm{~K}$, whereas it shifts to lower energies for higher temperatures. No change of the FWHM of the peak is appreciable within experimental error: Its value is $(0.85 \pm 0.08) \mathrm{eV}$. In $c-\mathrm{SiO}_{2}$ at $10 \mathrm{~K}$ the FWHM of the $10.5-\mathrm{eV}$ peak is $(0.69 \pm 0.04) \mathrm{eV}$. In the matter of the peak at $\sim 11.6 \mathrm{eV}$, its position and amplitude, or area, cannot be quantitatively determined due to its closeness to the two other peaks at $\sim 10.4$ and $\sim 14.1 \mathrm{eV}$, respectively.

The spectra in Fig. 1 cannot be used to directly evaluate the parameters of the Urbach tail that are typical of the $E$ $<9 \mathrm{eV}$ spectral region. In fact, the noise amplitude on the spectra in Fig. 1 is of the order of $10^{3} \mathrm{~cm}^{-1}$. Hence, K-K absorption spectra are reliable only for absorption coefficients larger than $\sim 10^{4} \mathrm{~cm}^{-1}$. While this is only $1 \%$ of the $10.4-$ $\mathrm{eV}$ peak amplitude and it does not affect any consideration concerning spectra for $E>9 \mathrm{eV}$, on the other hand, it means that the Urbach tail is concealed within experimental error on the K-K absorption spectrum, as usual for this kind of analysis. It is impossible, then, to analyze here the spectral region in which the Urbach tail joins the left wing of the excitonic peak, where $\alpha(E)$ is expected to feature an inflection point. For this reason, in Sec. IV B the properties of the $10.4-\mathrm{eV}$ peak will be compared with those of the Urbach tail as arising from previous studies. ${ }^{6,28,30}$

Photoluminescence emission spectra under excitation with synchrotron radiation were acquired in front-face geometry. The signal was dispersed by a 300 -lines/mm monochromator with 500-nm blaze, and detected by a charge-coupled camera cooled by liquid $\mathrm{N}_{2}$. The emission bandwidth was $10 \mathrm{~nm}$. The spectra were corrected for the response and dispersion of the

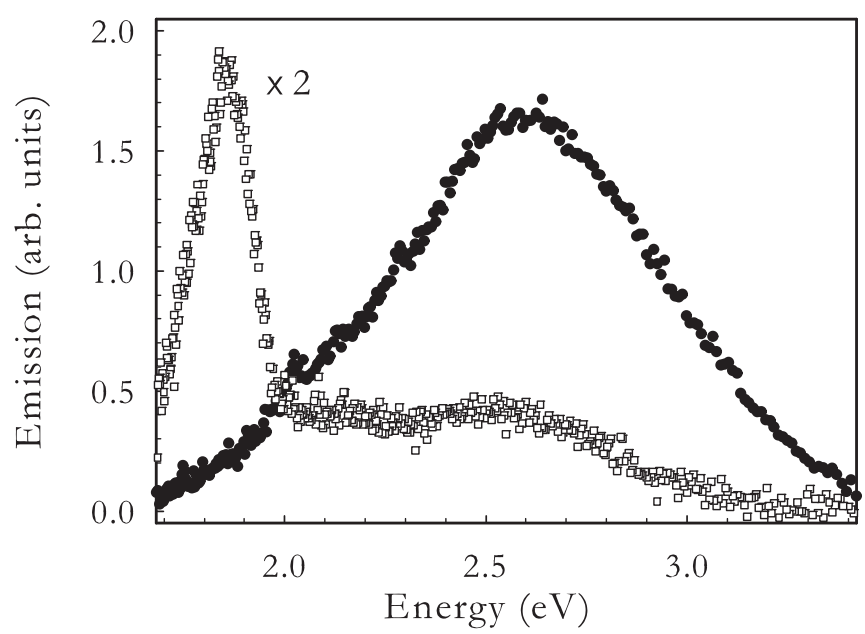

FIG. 4. Photoluminescence emission spectrum detected at $T=$ $10 \mathrm{~K}$ in $c-\mathrm{SiO}_{2}$ (circles) and $a-\mathrm{SiO}_{2}$ (squares) under excitation with synchrotron radiation of $10.2 \mathrm{eV}$ photon energy.

detection system. Figure 4 shows a typical photoluminescence emission spectrum detected at $T=10 \mathrm{~K}$ in $c-\mathrm{SiO}_{2}$ and $a-\mathrm{SiO}_{2}$ upon excitation at $10.2 \mathrm{eV}$, falling within the excitonic peak.

\section{A COMPREHENSIVE MODEL FOR THE INTERPRETATION OF OPTICAL FEATURES IN $\mathrm{SiO}_{2}$ FROM 8 TO $11 \mathrm{eV}$}

This section presents our model for the interpretation of experimental data on above-edge optical properties in $\mathrm{SiO}_{2}$ and is organized as follows. In Sec. IV A we introduce the mobility properties of excitons in $\mathrm{SiO}_{2}$ briefly summing up what we have already reported in a recent paper. ${ }^{27} \mathrm{In} \mathrm{Sec}$. IV B we analyze the relations existing between the features of the first excitonic peak and those of the Urbach absorption tail. Section IV C is devoted to providing estimates of the physical parameters governing exciton dynamics in $\mathrm{SiO}_{2}$. Section IV D deals with the comparison between our results and literature data on $\mathrm{SiO}_{2}$ and on other solid systems. Finally in Sec. IV E the properties of the peaks at energies higher than $10.4 \mathrm{eV}$ are discussed.

\section{A. Comparison with theoretical models: Exciton mobility in $\mathrm{SiO}_{2}$}

The K-K-derived absorption spectra reported in Fig. 1 agree qualitatively with those observed in other $a-\mathrm{SiO}_{2}$ and $c-\mathrm{SiO}_{2}$ samples and previously reported in literature. ${ }^{12}$ While it has been widely accepted for a long time that, in both $c-\mathrm{SiO}_{2}$ and $a$ $\mathrm{SiO}_{2}$, the 10.4-eV peak has an excitonic origin, ${ }^{10,12,15,19,21,25,26}$ we have only recently shown ${ }^{27}$ that this peak has a remarkably good Lorentzian line shape at all the examined temperatures. This circumstance bears a deep meaning within Toyozawa's theory of line shapes of excitonic absorption bands, in the limit of weak exciton-phonon scattering. ${ }^{31,33}$ First, this result allows to argue that the effect of structural disorder intrinsic to the amorphous $a-\mathrm{SiO}_{2}$ structure is qualitatively the same as that of increasing temperature, in that both lead to an increase of the root-mean-square amplitude $D$ of excitonic 
energy fluctuations (see Sec. II). Second and most important, the close Lorentzian line shape proves the mobile, delocalized nature of excitons in both $c-\mathrm{SiO}_{2}$ and $a-\mathrm{SiO}_{2}$. In other words, from 10 to $300 \mathrm{~K}$, the exciton mobility is so large as compared to the effects of both static disorder and EPC in $a-\mathrm{SiO}_{2}$ that the exciton band keeps its Lorentzian profile, not presenting any tendencies toward a Gaussian one even at the highest considered temperature $(300 \mathrm{~K})$. Being that exciton mobility is ultimately related to the translational symmetry of the lattice, this result leads to the remarkable conclusion that the lack of long-range order typical of an amorphous solid turns out to have a negligible effect on the properties of excitons, meaning that the effect of static disorder in $a-\mathrm{SiO}_{2}$ is low enough to closely preserve crystal-like delocalization of excitons. It appears that, at least at low temperatures, the difference between the excitonic properties of $a-\mathrm{SiO}_{2}$ and $c-\mathrm{SiO}_{2}$ comes down to a greater width of the first exciton absorption peak in the amorphous system, $(0.85 \pm 0.08) \mathrm{eV}$ instead of $(0.69 \pm 0.04) \mathrm{eV}$, likely due to structural disorder introducing small additional site-to-site fluctuations of the exciton energy contributing to $D$.

Let us now discuss the temperature dependence of the spectral features of the 10.4-eV peak. The position of the peak as a function of temperature is expected to be described by the equation $E_{P}(T)=E_{P}^{0}+\Delta_{0}(T), \Delta_{0}(T)$ being given by Eq. (2). In Fig. 3 the values of the peak positions as determined from the Lorentzian fittings of experimental spectra and the curve obtained by least-square fitting data with this equation are reported. As can be seen, Eq. (2) is consistent with the observed trend, with the only possible exception of the point at $T=10 \mathrm{~K}$. Admittedly the present results do not allow to establish if the observed scatter of this datum points out a low-temperature deviation from the accepted theoretical description as far as the temperature dependence of the peak is concerned. The best fitting values of the parameters are $E_{P}^{0}=(10.79 \pm 0.05) \mathrm{eV}, \Delta_{0}^{0}=(-0.38 \pm 0.05) \mathrm{eV}$, and $\hbar \omega_{0}=(0.083 \pm 0.002) \mathrm{eV}$ and are reported in Table I.

\section{B. Relation with the Urbach absorption tail}

In the framework of Toyozawa's theory the characteristic Urbach exponential absorption tail (UT) given by Eq. (3) has been predicted to be a typical feature existing on the left wing of Lorentzian exciton peaks as a consequence of the creation of momentary self-trapped excitons. ${ }^{35,36}$ Thus, on a qualitative basis, the present findings on the delocalized nature of the 10.4-eV exciton agree with the characteristic presence in $\mathrm{SiO}_{2}$ of the UT as observed by absorption measurements

TABLE I. Exciton-related parameters extracted from the temperature dependence of the 10.4-eV excitonic peak in $a-\mathrm{SiO}_{2}$ (upper section). Proposed estimates of the parameters defined in Sec. II controlling exciton dynamics in $a-\mathrm{SiO}_{2}$ (lower section). Correspondent values for $c-\mathrm{SiO}_{2}$ are reported in the text.

\begin{tabular}{lccccc}
\hline \hline & $\hbar \omega_{0}(\mathrm{eV})$ & $E_{P}^{0}(\mathrm{eV})$ & $\Delta_{0}^{0}(\mathrm{eV})$ & \\
\hline & 0.083 & 10.79 & -0.38 & \\
$B(\mathrm{eV})$ & $D(\mathrm{eV})$ & $D / B$ & $E_{\mathrm{LR}}(\mathrm{eV})$ & $g$ & $\eta(10.2 \mathrm{eV})$ \\
$\sim 2$ & $\sim 0.7$ & $\sim 0.35$ & $\sim 4$ & 2.1 & $\sim 10^{-3}$ \\
\hline \hline
\end{tabular}

between 8 and $9 \mathrm{eV}^{29,30}$ Furthermore, the present conclusions on the negligible influence of amorphous disorder on the 10.4-eV peak are consistent with previous investigations on the effect of structural disorder on the properties of the UT in $a-\mathrm{SiO}_{2}$ showing that, if compared to thermal disorder, structural disorder accounts for a minor portion only of the broadening of the intrinsic edge. ${ }^{29,30}$

The agreement becomes much stronger when one notices that the value of the parameter $\hbar \omega_{0}$ we obtained from the temperature dependence of the $10.4-\mathrm{eV}$ peak is in a very good agreement with that already available in literature ${ }^{29}$ for $a-\mathrm{SiO}_{2}, \hbar \omega_{0}=(0.089 \pm 0.008) \mathrm{eV}$, deduced from the study of the temperature dependence of the UT. (This value was obtained from UT data on $a-\mathrm{SiO}_{2}$ samples featuring a low $\mathrm{Si}-\mathrm{OH}$ impurity content and may be slightly different in other types of $a-\mathrm{SiO}_{2}$. However, the comparison is fully meaningful as the $a-\mathrm{SiO}_{2}$ type used in the present study coincides with that in which $\hbar \omega_{0}$ was determined in Ref. 29.) This strongly supports Toyozawa's interpretation of the UT being a low-energy feature peculiar of a weakly scattered exciton absorption peak ${ }^{33,35}$ and its variations as a function of temperature as a consequence of those of the first exciton peak. In this context it should be noted that, as far as the UT is concerned, $a-\mathrm{SiO}_{2}$ is not representative of the behavior of all amorphous systems. Indeed, a "glassy" variant of the UT is observed in several amorphous solids, ${ }^{38,39}$ where the slope of the exponential profile in a semilogarithmic scale, the so-called Urbach energy, does not depend on temperature in contrast with Eqs. (3) and (4). Thus, the fact that, notwithstanding its amorphous structure, $a-\mathrm{SiO}_{2}$ shows the standard Urbach rule [Eqs. (3) and (4)] further indicates that structural disorder hardly affects the electronic properties of the material.

The association of the UT to the 10.4-eV peak in $\mathrm{SiO}_{2}$ has been questioned, ${ }^{28}$ based on the position of the temperatureindependent crossing point $\left(E_{0}, \alpha_{0}\right)$ of the UTs, which is expected to exist as a consequence of Eq. (3). Indeed, experimental investigations have found the spectral position of the crossing point to be located at $E_{0}=9.1 \mathrm{eV}$ in $c-\mathrm{SiO}_{2}$ and at $E_{0}=8.7 \mathrm{eV}$ in $a-\mathrm{SiO}_{2}{ }^{28}$ Based on the difference between $E_{0}$ and the position $10.4 \mathrm{eV}$ of the exciton peak, it was proposed that the exciton responsible for the $10.4-\mathrm{eV}$ peak is different from that associated with the UT. ${ }^{21,28}$ However, the convergence of the UTs close to the energy position of the main peak is expected only when the energy gap between the valence and conduction bands is direct, as in alkali halides. ${ }^{32,35}$ In contrast, if the gap is indirect (i.e., the $K=0$ exciton level is not at the bottom of the exciton band) the excitonic peak moves to a higher energy, while the crossing point of the UTs is still expected to stay at the energy corresponding to the bottom of the exciton band, i.e., recalling that the width of the exciton energy band is $2 B$ (see Sec. II), an energy $B$ below the band center. $^{32,35}$ Thus, in the simplest case of a $K=0$ level located at the center of the band, the distance between the converging point and the excitonic peak is $B$. From an experimental point of view this is the situation found, for instance, in $\mathrm{AgCl}$, where $E_{0}=3.3 \mathrm{eV}$ corresponds to the indirect band gap of the material, while the excitonic peak is at $5.0 \mathrm{eV} .^{31,35,40}$

Then, for $a-\mathrm{SiO}_{2}$ there is no conflict between $E_{0}=8.7 \mathrm{eV}$ and the excitonic peak at $10.4 \mathrm{eV}$ : This disagreement simply leads to conclude that $\mathrm{SiO}_{2}$ features an indirect gap near $9 \mathrm{eV}$ 
while the direct gap is above the value of $E_{P}^{0}$ found here, i.e., close to $\sim 11 \mathrm{eV}$. (The separation between the direct gap and $E_{P}^{0}$ equals the exciton binding energy.) The indirect nature of the gap in $\mathrm{SiO}_{2}$ is also consistent with results obtained with theoretical calculations. ${ }^{23,24}$ Based on these considerations, we can roughly estimate $B$ as the separation in energy between $E_{0}$ and $E_{P}^{0}$, i.e., $B \sim 10.8-8.7=2.1 \mathrm{eV}$. While this estimate applies to the simplest case of the $K=0$ level at the center of the exciton band, a lower threshold for $B$ can be obtained if one considers the case of $K=0$ at the top of the band, and is half of the just calculated value.

\section{Parameters affecting exciton dynamics}

A comparison between the present data and the features of the UT in $a-\mathrm{SiO}_{2}$ allowed us to estimate the value $B \sim$ $2.1 \mathrm{eV}$. Another totally independent line of reasoning leads to a consistent estimate of the magnitude of the same parameter. Several studies on the UT have led to estimate the parameter [see Eq. (3)] $\sigma_{0} \sim 0.5$ for $a-\mathrm{SiO}_{2}$ and $\sim 0.6$ for $c-\mathrm{SiO}_{2}{ }^{22}$ As explained in the Introduction, $\sigma_{0}$ is basically the inverse of the EPC constant $g=E_{\mathrm{LR}} / B$, leading to $g \sim 2$ in $a-\mathrm{SiO}_{2}$. Now, the parameter $E_{\mathrm{LR}}$, defined in Sec. II as the relaxation energy within the excited electronic state of the exciton, can be estimated approximately as the half Stokes shift between exciton absorption and emission. Since STE emission is at $\sim 2.5 \mathrm{eV}$ for $a-\mathrm{SiO}_{2}$ and at $2.6 \mathrm{eV}$ for $c-\mathrm{SiO}_{2}$ (Refs. 17, 21, and 22) [singlet and triplet emission being separated by a fraction of eV (Ref. 41)], we get $E_{\mathrm{LR}} \sim(10.4-2.5) / 2 \sim 4 \mathrm{eV}$ in $a-\mathrm{SiO}_{2}$. Hence, from $g=E_{\mathrm{LR}} / B$ we finally obtain $B \sim$ $2 \mathrm{eV}$. The good agreement with the previously estimated $B \sim$ $2.1 \mathrm{eV}$ strongly confirms the applicability of the whole line of reasoning. Similarly in $c-\mathrm{SiO}_{2}$ we get $B \sim 2.4 \mathrm{eV}, E_{\mathrm{LR}}=$ $4 \mathrm{eV}$. In the following we will assume $B \sim 2 \mathrm{eV}$ both in $a-\mathrm{SiO}_{2}$ and in $c-\mathrm{SiO}_{2}$. The proposed estimates of the parameters $B$, $E_{\mathrm{LR}}$, and $g$ are reported in Table I.

We are now in a position to provide and discuss approximate estimates of all the parameters controlling exciton dynamics. Starting from our estimation of the $B$ values, one can evaluate $D$ from the observed FWHM of the bands. In fact, the first-order theoretical expression FWHM $=\pi D^{2} / B$ (see Sec. II) can be inverted to get $D \sim 0.74 \mathrm{eV}$ in $a-\mathrm{SiO}_{2}$ $(\mathrm{FWHM}=0.85 \mathrm{eV})$ and $D \sim 0.66 \mathrm{eV}$ in $c-\mathrm{SiO}_{2}(\mathrm{FWHM}=$ $0.69 \mathrm{eV})$. The similarity between these two values confirms that the contribution of amorphous structural disorder to excitonic energy fluctuations is very poor, as anticipated. In the following we will consider $D \sim 0.7 \mathrm{eV}$ in both systems, leading to $D / B \sim 0.35$. The estimated values are reported in Table I. While, strictly speaking, this value of $D / B$ cannot be considered as $\ll 1$, it anyway most assuredly falls into the weak scattering category, also considering the fact that the transition between weak and strong scattering is a gradual one. Fluctuations $(D)$ of the exciton energy of the order of $0.7 \mathrm{eV}$ actually imply a rather efficient coupling of excitons with vibrational modes, being that the weak scattering condition is anyway ensured by the large extension of the excitonic band. This is the situation referred to as strong EPC ( $g \sim 2$ ) and weak scattering $(D / B \sim 0.35)$, characterized by an excitonic peak which is relatively wide (since FWHM $\propto$ $D^{2}$ ) yet Lorentzian and by STE-type emission, as expected when $g>1$ and as observed in $\mathrm{SiO}_{2}$. It should be noted here that another estimate of $D$ can be obtained via Eq. (2) in the limit $T \rightarrow 0$ by using $E_{\mathrm{LR}} \sim 4 \mathrm{eV}$ and $\hbar \omega_{0}=0.083 \mathrm{eV}$ found before. This yields $D \sim 0.6 \mathrm{eV}$ in $a-\mathrm{SiO}_{2}$ at low temperatures. The good agreement with the previously calculated value $D \sim 0.7 \mathrm{eV}$ further strengthens our conclusions, especially if one considers that the latter was inferred by an expression for the Lorentzian FWHM which represents only a first-order approximation.

Being that the absorption energy is as high as $10.4 \mathrm{eV}$, a value of $E_{\mathrm{LR}}$ of the order of a few $\mathrm{eV}$ is reasonable in the scheme of strong EPC. On the other hand, by applying a Dexter criterion for luminescence, ${ }^{34}$ the value of $E_{\mathrm{LR}} \sim$ $4 \mathrm{eV}$ should be associated with a significant probability of nonradiative exciton decay, typically leading to the creation of point defects. ${ }^{1,2}$ This is consistent with the experimentally observed emission properties of $\mathrm{SiO}_{2}$, where nonradiative decay of excitons has been widely recognized as an important mechanism for the photoinduced generation of defects. ${ }^{1,2}$ In this respect, it is useful to consider data reported in Fig. 4, showing a typical photoluminescence emission spectrum detected at $T=10 \mathrm{~K}$ in $c-\mathrm{SiO}_{2}$ and $a-\mathrm{SiO}_{2}$ upon excitation at $10.2 \mathrm{eV}$, falling within the excitonic peak. The signal observed in $c-\mathrm{SiO}_{2}$ consists of a wide band peaked at $2.60 \mathrm{eV}$, which is the well-known emission due to the STE. The signal in $a-\mathrm{SiO}_{2}$ is composite: The broad band centered at $2.5 \mathrm{eV}$ is due to STE luminescence, while the 1.9-eV peak is due to the point defect known as the nonbridging oxygen hole center, ${ }^{2}$ generated at low temperatures in $a-\mathrm{SiO}_{2}$ by synchrotron radiation. ${ }^{42}$ From data in Fig. 4 we were able to estimate the excitonic emission quantum yield $\eta$ at $T=10 \mathrm{~K}$ by comparing the intensity of the signal with that of a system of known quantum yield (sodium salycilate, ${ }^{43} \eta=0.5$ ) measured in the same experimental conditions. In this way, we estimated $\eta\left(c-\mathrm{SiO}_{2}\right) \sim 10^{-2}$ and $\eta\left(a-\mathrm{SiO}_{2}\right) \sim 10^{-3} \mathrm{~T}=10 \mathrm{~K}$ under excitation at $10.2 \mathrm{eV}$. These values are $\ll 1$ as expected.

\section{Comparison with literature on $\mathrm{SiO}_{2}$ and other systems}

The strong experimental evidence that the peak is accurately described by a simple Lorenztian function seriously casts doubt on the model widely accepted in $\mathrm{SiO}_{2}$ literature, claiming a strong localization and consequently a low mobility of excitons in $a-\mathrm{SiO}_{2} \cdot{ }^{21,22}$ Such a model, based on the study of the energy-transfer processes from excitons to luminescent impurities, described the $10.4-\mathrm{eV}$ band, even in $c-\mathrm{SiO}_{2}$, as the overlap of several Gaussian subbands associated with several types of localized excitons. Aside from the papers supporting this model, the other existing literature on $\mathrm{SiO}_{2}$ did not address the issue of the line shape of the 10.4-eV peak, likely due to an insufficient quality of data or to their work being focused on other aspects. It is worth considering here data reported by one of the most recent studies of above-edge absorption in $\mathrm{SiO}_{2}$, where measurements were carried out by using a VUV laser plasma light source. ${ }^{12}$ By carrying out a (not reported) analytical fitting procedure on the absorption spectrum reported for $c-\mathrm{SiO}_{2}$, as acquired from the published paper, we found that the reported $10.5-\mathrm{eV}$ peak in $c-\mathrm{SiO}_{2}$ has a satisfactory Lorentzian profile as well, although the FHWM turns out to be slightly larger $(\mathrm{FWHM}=0.88 \mathrm{eV})$ and small 
deviations appear in the region below $9.3 \mathrm{eV}$. With regard to $a-\mathrm{SiO}_{2}$, published data do not contrast with our findings, although the signal-to-noise ratio is insufficient to conclusively determine the line shape of the peak.

Lorentzian exciton line shapes have been reported in literature for several crystalline systems at low temperatures. ${ }^{44,45}$ Although most of them actually feature very sharp absorption and free-exciton photoluminescence emission lines, a few examples exist featuring relatively wide yet Lorentzian exciton line shapes. These are systems featuring a strong EPC but weak scattering (as proposed here for $\mathrm{SiO}_{2}$ ), ${ }^{33,34}$ and basically consist of wide-band-gap crystals such as $\mathrm{LiF}$ and $\mathrm{NaF}$. For instance, the first excitonic absorption in $\mathrm{LiF}$ and $\mathrm{NaF}$ have been reported to be Lorentzians peaked at 12.6 and $10.7 \mathrm{eV}$ with 0.4 and $0.12 \mathrm{eV}$ widths, respectively. ${ }^{46,47}$ (The peak in $\mathrm{NaF}$ is actually a doublet composed of two Lorentzian shapes. The 0.12-eV FWHM quoted in the text is the width of each of the two components.) The analogies between $\mathrm{SiO}_{2}$ and these other very-wide-band-gap systems also extend to excitonic photoluminescence properties and to the properties of the far low-energy tail of the excitonic peak. In fact, also LiF and $\mathrm{NaF}$ feature wide Stokes-shifted STE luminescence bands [e.g., emission at $3.4 \mathrm{eV}$ for $\mathrm{LiF}$ (Ref. 48)], thus being consistent with the emission properties of $\mathrm{SiO}_{2}$ upon above-edge excitation. Furthermore, $\mathrm{LiF}$ and $\mathrm{NaF}$ also feature the UT on the left wing of their Lorentzian absorption peaks. ${ }^{46,49}$ Thus, $\mathrm{LiF}$ and $\mathrm{NaF}$ can be classified, as $\mathrm{SiO}_{2}$, as featuring strong coupling to, but weak scattering by, phonons.

Excitonic absorption bands in $\mathrm{LiF}$ and $\mathrm{NaF}$ feature a certain degree of asymmetry which is apparently not observed here for $\mathrm{SiO}_{2}$. Theory predicts asymmetry properties to depend on the position of the $K=0$ level inside the exciton band. ${ }^{31}$ If the $K=0$ level is at the center of the band, the resulting line shape is predicted to be symmetric, while if the $K=0$ level is in the upper or lower half of the band, the line shape is expected to be steeper on the right (negative asymmetry) or left (positive asymmetry) side, respectively. ${ }^{31}$ The last case includes the situation of a direct band gap (as in $\mathrm{LiF}$ and $\mathrm{NaF}$ ), for which the Lorentzian description is most accurate on the right half of the peak. Actual systems showing Lorentzian profiles with both positive, negative, and very low asymmetry were observed in literature. For instance, $\mathrm{AgCl}$ (indirect gap at $3.3 \mathrm{eV}$ ) features an excitonic peak at $5.0 \mathrm{eV}$ with $0.3-\mathrm{eV}$ FWHM which is Lorentzian on the left side, while on the right side the overlap with other bands prevent an accurate analysis, ${ }^{31,35,40}$ which is a very similar situation as that observed here for $\mathrm{SiO}_{2}$. Thus, in addition to the distance between the crossing point of the UTs and the excitonic peak, also the lack of asymmetry suggests $\mathrm{SiO}_{2}$ to feature an indirect band gap, and, in particular, the $K=0$ level to be close to the center of the exciton band. On the other side, we cannot rule out the possibility of a steeper high-energy wing of the exciton peak, as this hidden by the superposition with the $11.5-\mathrm{eV}$ peak, which would lead to the $K=0$ level being in the upper half of the excitonic band.

As far as the influence of the amorphous nature of the material is concerned, it is worth noting that the possibility of delocalized excitons was tentatively proposed in other systems, such as $a-\mathrm{As}_{2} \mathrm{~S}_{3},{ }^{50}$ based on indirect evidence arising from luminescence measurements. On the other side, the behavior of $\mathrm{SiO}_{2}$ is deeply different from that of other materials such as $\mathrm{Si}$, where a dramatic change in the shape and broadening of the excitonic peak at $3.5 \mathrm{eV}$ are observed when going from the crystal to the amorphous solid. ${ }^{51}$ Thus, it cannot be taken for granted for all systems that amorphous disorder introduces only a small perturbation of the delocalized exciton states.

\section{E. Suggestions for the higher-energy peaks}

While there is general agreement on the fact that the $\sim 14.1$ and $\sim 17.3 \mathrm{eV}$ peaks are associated with maxima of the interband transition cross section, ${ }^{12}$ as to the $\sim 11.6 \mathrm{eV}$ peak, several authors suggested the presence of two or more contributions both peaking in this energy region, only one of which is associated with excitonic absorption, ${ }^{15,18,23}$ possibly related to the first excited state of the $10.4-\mathrm{eV}$ exciton. ${ }^{18}$ As for the region of the interband transition peaks, our data in Fig. 1 indicate that the absorption coefficient at $E>$ $12.2 \mathrm{eV}$ slightly increases as a whole without changing its shape from 10 to $150 \mathrm{~K}$. While we acknowledge that the present results do not allow to clarify the nature of this increase, it certainly has consequences on the apparent temperature dependence of the $\sim 11.6 \mathrm{eV}$ structure. As a matter of fact, a comparison between Figs. 2(c) and 1 clearly reveals that the apparent variations of the peak at $\sim 11.6 \mathrm{eV}$ from 150 to $10 \mathrm{~K}$ are only a consequence of those observed in the remaining higher-energy part of the spectra. On the other hand, in the range of temperatures from 300 to $150 \mathrm{~K}$ the observed decrease [Fig. 2(b)] of the amplitude of the peak at $\sim 11.6 \mathrm{eV}$ is peculiar to this structure only: Indeed, it can be clearly singled out from the temperature-induced modifications of the higher-energy side of the spectra, since the high- and low-energy wings of the band do not change with varying temperature from 300 to $150 \mathrm{~K}$. While a quantitative analysis of the temperature dependence of the $\sim 11.6 \mathrm{eV}$ peak is hindered by its overlap with the nearby structures, an analogous decrease for $T>$ $150 \mathrm{~K}$ is not observed for the higher-energy peaks at $\sim 14.1$ and $\sim 17.3 \mathrm{eV}$. Based on these qualitative differences we suggest that, in $a-\mathrm{SiO}_{2}$, the peak at $\sim 11.6 \mathrm{eV}$ and those at higher energies do not share a common physical origin.

\section{CONCLUSIONS}

A model for the comprehensive description of the optical features in the range from $\sim 8$ up to $\sim 11 \mathrm{eV}$ is proposed. Our model is founded on the analysis of Kramers-Kronig-derived absorption spectra in the range from $\sim 8$ up to $\sim 17.5 \mathrm{eV}$, together with the features of the Urbach absorption tail and of the self-trapped exciton emission. Our description significantly improves the current understanding of excitonic properties of $\mathrm{SiO}_{2}$. The study of the temperature dependence of the position of the excitonic peak at $\sim 10.4 \mathrm{eV}$ allowed to determine the value of the average vibrational energy of phonons interacting with excitons $\left[\hbar \omega_{0}=(0.083 \pm\right.$ $0.002) \mathrm{eV}$. The properties of the Urbach exponential absorption tail and those of self-trapped exciton photoluminescence excited above gap are qualitatively and quantitatively coherent with those of the 10.4-eV absorption peak. This allows to interpret self-consistently the whole set of optical properties related to excitation of $a-\mathrm{SiO}_{2}$ from $\sim 8$ to $\sim 11 \mathrm{eV}$ as determined by excitons, demonstrates the 
applicability of Toyozawa's theory for excitonic line shapes to an amorphous system and supports the interpretation of the Urbach tail as resulting from momentary self-trapping of excitons. Moreover, our data provide estimates of the main theoretical parameters governing exciton-phonon interaction, such as the half width of the excitonic energy band ( $B \sim 2 \mathrm{eV}$ ), the root-mean-square amplitude of site-to-site energy fluctuations of exciton energy ( $D \sim 0.7 \mathrm{eV})$, and the exciton-phonon coupling constant $(g \sim 2.1)$. The quantum yield of the excitonic emission $\left(\eta \sim 10^{-3}\right)$ at $T=10 \mathrm{~K}$ was determined, too. Exciton dynamics turns out to be satisfactorily described in the context of a strong exciton-phonon coupling coexisting with weak exciton-phonon scattering, with $\mathrm{SiO}_{2}$ featuring an indirect band gap at $\sim 9 \mathrm{eV}$ and a threshold for direct band-to-band transitions at $\sim 11 \mathrm{eV} . a-\mathrm{SiO}_{2}$ and $c-\mathrm{SiO}_{2}$ are found to be very similar with regard to exciton properties, apart from a small additional contribution to the excitonic energy fluctuations in the amorphous case due to disorder.

\section{ACKNOWLEDGMENTS}

We acknowledge financial support from DESY, and we thank the LAMP group (http://www.fisica.unipa.it/ amorphous) for useful and stimulating discussions. *fmessina@ fisica.unipa.it

${ }^{1}$ Silicon-Based Materials and Devices, edited by H. S. Nalwa (Academic, New York, 2001).

${ }^{2}$ L. Skuja, H. Hosono, and M. Hirano, Proc. SPIE 4347, 155 (2001).

${ }^{3}$ L. Skuja, H. Hosono, M. Hirano, and K. Kajihara, Proc. SPIE 5122, 2 (2003).

${ }^{4}$ K. Kajihara, J. Ceram. Soc. Jpn. 115, 85 (2007).

${ }^{5}$ E. K. Chang, M. Rohlfing, and S. G. Louie, Phys. Rev. Lett. 85, 2613 (2000).

${ }^{6}$ E. Vella, R. Boscaino, and G. Navarra, Phys. Rev. B 77, 165203 (2008).

${ }^{7}$ H. R. Philipp, Solid State Commun. 4, 73 (1966).

${ }^{8}$ H. R. Philipp, J. Phys. Chem. Solids 32, 1935 (1971).

${ }^{9}$ P. L. Lamy, Appl. Opt. 16, 2212 (1977).

${ }^{10}$ C. Bosio and W. Czaja, Europhys. Lett. 24, 197 (1993).

${ }^{11}$ G.-L. Tan, M. F. Lemon, and R. H. French, J. Am. Ceram. Soc. 86, 1885 (2003).

${ }^{12}$ G. L. Tan, M. F. Lemon, D. J. Jones, and R. H. French, Phys. Rev. B 72, 205117 (2005).

${ }^{13}$ Z. A. Weinberg, G. W. Rubloff, and E. Bassous, Phys. Rev. B 19, 3107 (1979).

${ }^{14}$ T. H. DiStefano and D. E. Eastman, Solid State Commun. 9, 2259 (1971).

${ }^{15}$ K. Platzöder, Phys. Status Solidi 29, K63 (1968).

${ }^{16}$ R. B. Laughlin, Phys. Rev. B 22, 3021 (1980).

${ }^{17}$ C. Itoh, K. Tanimura, N. Itoh, and M. Itoh, Phys. Rev. B 39, 11183 (1989).

${ }^{18}$ C. Bosio, W. Czaja, and H. C. Mertins, Europhys. Lett. 18, 319 (1992).

${ }^{19}$ Y. M. Alexandrov, V. M. Vishnjakov, V. N. Makhov, K. K. Sidorin, A. N. Thrukhin, and M. N. Yakimenko, Nucl. Instrum. Methods Phys. Res., Sect. A 282, 580 (1989).

${ }^{20}$ R. Evrard and A. N. Trukhin, Phys. Rev. B 25, 4102 (1982).

${ }^{21}$ A. N. Trukhin, J. Non-Cryst. Solids 149, 32 (1992).

${ }^{22}$ A. N. Trukhin, in Defects in $\mathrm{SiO}_{2}$ and Related Dielectrics: Science and Technology, edited by G. Pacchioni, L. Skuja, and D. L. Griscom (Kluwer Academic, New York, 2000), p. 235.

${ }^{23}$ J. R. Chelikowski and M. Schlüter, Phys. Rev. B 15, 4020 (1977).

${ }^{24}$ N. F. Mott, in The Physics of $\mathrm{SiO}_{2}$ and Its Interfaces, edited by S. T. Pantelides (Pergamon, New York, 1978), p. 80.
${ }^{25}$ E. Loh, Solid State Commun. 2, 269 (1964).

${ }^{26}$ O. M. Sorokin and V. A. Blank, Opt. Spectrosc. 41, 602 (1976).

${ }^{27}$ F. Messina, E. Vella, M. Cannas, and R. Boscaino, Phys. Rev. Lett. 105, 116401 (2010).

${ }^{28}$ I. Godmanis, A. N. Trukhin, and K. Hübner, Phys. Status Solidi 116, 279 (1983).

${ }^{29}$ K. Saito and A. J. Ikushima, Phys. Rev. B 62, 8584 (2000).

${ }^{30}$ E. Vella and R. Boscaino, Phys. Rev. B 79, 085204 (2009).

${ }^{31}$ Y. Toyozawa, Prog. Theor. Phys. 20, 53 (1958).

${ }^{32}$ Y. Toyozawa, Optical Processes in Solids (Cambridge University Press, Cambridge, UK, 2003).

${ }^{33}$ M. Schreiber and Y. Toyozawa, J. Phys. Soc. Jpn. 51, 1528 (1982).

${ }^{34}$ Y. Toyozawa, Pure Appl. Chem. 69, 1171 (1997).

${ }^{35}$ H. Sumi and Y. Toyozawa, J. Phys. Soc. Jpn. 31, 342 (1971).

${ }^{36}$ M. Schreiber and Y. Toyozawa, J. Phys. Soc. Jpn. 51, 1544 (1982).

${ }^{37}$ J. Tauc, in Amorphous and Liquid Semiconductors, edited by J. Tauc (Plenum, New York, 1974), p. 159.

${ }^{38}$ A. N. Trukhin and P. Kulis, J. Non-Cryst. Solids 188, 125 (1995).

${ }^{39}$ I. A. Weinstein and A. F. Zatsepin, Phys. Status Solidi C 1, 2916 (2004).

${ }^{40}$ S. Tutihasi, Phys. Rev. 105, 882 (1957).

${ }^{41}$ A. N. Trukhin, J. Phys. Condens. Matter 20, 125217 (2008).

${ }^{42}$ F. Messina, L. Vaccaro, and M. Cannas, Phys. Rev. B 81, 035212 (2010).

${ }^{43}$ A. N. Trukhin, M. Kink, Y. Maksimov, and R. Kink, Solid State Commun. 127, 655 (2003).

${ }^{44}$ T. Tomiki, T. Miyata, and H. Tsukamoto, J. Phys. Soc. Jpn. 35, 495 (1973).

${ }^{45}$ D. M. Burland, U. Konzelmann, and R. M. Macfarlane, J. Chem. Phys. 67, 1926 (1977).

${ }^{46}$ R. Sano, J. Phys. Soc. Jpn. 27, 695 (1969).

${ }^{47}$ M. Piacentini, Solid State Commun. 17, 697 (1975).

${ }^{48}$ S. Nakonechnyi, T. Kärner, A. Lushchik, C. Lushchik, V. Babin, E. Feldbach, I. Kudryavtseva, P. Liblik, L. Pung, and E. Vasilchenko, J. Phys. Condens. Matter 18, 379 (2006).

${ }^{49}$ T. Tomiki and T. Miyata, J. Phys. Soc. Jpn. 27, 658 (1969).

${ }^{50}$ G. S. Higashi and M. Kastner, Phys. Rev. Lett. 47, 124 (1981).

${ }^{51}$ D. E. Aspnes, A. A. Studna, and E. Kinsbron, Phys. Rev. B 29, 768 (1984). 